\title{
Semantic Web Portal in University Research Community Framework
}

\author{
Rahmat Hidayat, Yazrina Yahya, Shahrul Azman Mohd Noah, \\ Mohd Zakree Ahmad, Abdul Razak Hamdan \\ Faculty of Information Science \& Technology, Universiti Kebangsaan Malaysia, Selangor Malaysia \\ E-mail: \{rahmat, yaz, samn, zakree,arh\}@ftsm.ukm.my
}

\begin{abstract}
One way overcome the weakness of semantic web to make it more user friendly is by displaying, browsing and semantically query data. In this research, we propose Semantic Web Research Community Portal at Faculty of Information Science and Technology - Universiti Kebangsaan Malaysia (FTSM RC) as the lightest platform of Semantic Web. This platform assists the users in managing the content and making visualization of relevant semantic data by applying meaningful periodically research. In such a way it will strengthen the research information related to research, publications, departments, organizations, events, and groups of researchers. Moreover, it will streamline the issuance process, making it easier for academic staff, support staff, and faculty itself to publish information of faculty and studies research information. By the end, this will provide end users with a better view of the structure of research at the university, allowing users to conduct cross-communication between faculty and study groups by using the search information.
\end{abstract}

Keywords—reseach community; semantic web; visualization

\section{INTRODUCTION}

The Internet is a technology that brings great changes in human life. Since the idea of the World Wide Web by Tim Berners-Lee [1] has been triggered, the development of the Internet has reached almost all regions all over the world. It has subjected and enhanced people capability to carry out their activities. In this technological age when people have email, use Internet banking services, have transactions using e-commerce, and even make connections in social networks on the Internet.

The diversity of applications arising from Internet development has increased the number of Internet users and reached widespread usage [2]. Statistics from internetworldstats.com shows the utilization of the Internet has reached almost $28.72 \%$ of the world's population (about 6.8 billion people). This amount represents the composition of $42 \%$ of the population from Asia, $24 \%$ of the population of the European continent, 13\% from North America, 10\% from Latin America and the rest of the population came from Africa and Oseania [2]..

The increasing number of Internet users is resulting in the number of web pages stored up very rapidly. This increasing number hus close relationship with blog technology that simplifies its users to create a web page. According to a study by NETCRAFT [3], the number of web pages available on the Internet in February 2007 amounted to 29.7 billion pages. The increase also occurred in the number of files stored on the Internet, such as papers, journals and conferences. Using e-mail with an attachment, a network of peer-to-peer networking, forums and personal web page also play a role in the larger and the number of files stored on the Internet. The increase Total files and web pages can cause difficulty for the user to find information on the internet [4].

Nowadays, the human scientific knowledge is stored in millions of papers, among 10,000 database which are rarely connected to each other, 1.000 sources of software that is not the search facility, and certainly in the head or thinking experts in the around the world and the whole landscape of science [5].

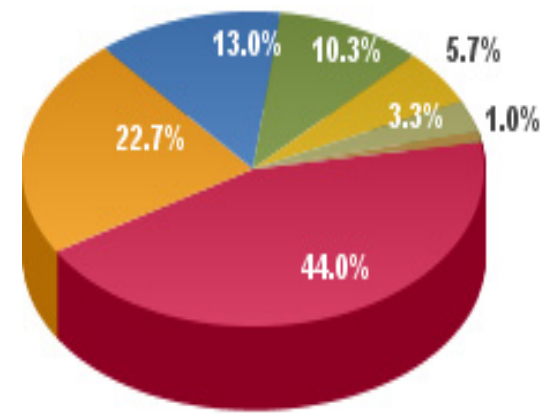

Asia $\mathbf{4 4 . 0 \%}$ Europe $22.7 \%$ north America 13.0\% Lat Am / Caribb $10.3 \%$ Africa $\mathbf{5 . 7 \%}$ Middle East 3.3\% Oceania / Australia $1.0 \%$

Fig. 1 Distribution of Internet users Based on Continent (source : Internet World Stats - www.internetwolrdstats.com/stats.htm) 
TABLE I

CONSUMER INTERNET AND THE WORLD POPULATION AT MARCH 31, 2011

\begin{tabular}{|c|c|c|c|c|c|c|}
\hline World Regions & $\begin{array}{c}\text { Population } \\
\text { (2011 Est. })\end{array}$ & $\begin{array}{c}\text { Internet Users } \\
\text { Dec. 31, 2000 }\end{array}$ & $\begin{array}{c}\text { Internet Users } \\
\text { Latest Data }\end{array}$ & $\begin{array}{c}\text { Penetration } \\
\text { (\% Population) }\end{array}$ & $\begin{array}{c}\text { Growth } \\
\mathbf{2 0 0 0 - 2 0 1 1}\end{array}$ & $\begin{array}{c}\text { Users \% } \\
\text { of Table }\end{array}$ \\
\hline Africa & 1.037 .524 .058 & 4.514 .400 & 118.609 .620 & $11.4 \%$ & $2.527,4 \%$ & $5.7 \%$ \\
\hline Asia & 3.879 .740 .877 & 114.304 .000 & 922.329 .554 & $23.8 \%$ & $706,9 \%$ & $44.0 \%$ \\
\hline Europe & 816.426 .346 & 105.096 .093 & 476.213 .935 & $58.3 \%$ & $353,1 \%$ & $22.7 \%$ \\
\hline Middle East & 216.258 .843 & 3.284 .800 & 68.553 .666 & $31.7 \%$ & $1.987,0 \%$ & $3.3 \%$ \\
\hline North America & 347.394 .870 & 108.096 .800 & 272.066 .000 & $78.3 \%$ & $151,7 \%$ & $13.0 \%$ \\
\hline Latin America/Carib. & 597.283 .165 & 18.068 .919 & 215.939 .400 & $36.2 \%$ & $1.037,4 \%$ & $10.3 \%$ \\
\hline Oceania / Australia & 35.426 .995 & 7.620 .480 & 21.293 .830 & $60.1 \%$ & $179.4 \%$ & $1.0 \%$ \\
\hline WORLD TOTAL & 6.930 .055 .154 & 360.985 .492 & 2.095 .006 .005 & $30.2 \%$ & $480.4 \%$ & $100.0 \%$ \\
\hline
\end{tabular}

(source : Internet World Stats - www.internetwolrdstats.com/stats.htm)

\section{LITERATURE REVIEW}

Exchangine data, information, and knowledge is an activity that will occur between people who communicate each other, either verbally or using the media. Web is an effective media to use in exchange. Fast and easy access that is supported by information technology to make the web an important tool in communication as well as a source of information. Besides providing a very substantial amount of information, the Web also has a variety of information. Diversity or heterogeniti develops in syntax, structure, or semantics. It is coupled with the diversity of languages and domain information [6].

In addition to the number of ontology for semantic web is increasing a lot. Similarly, the number of ontology repository that collects ontology, for example, Swoogle [7], Watson [8], OntoSelect [9] and SWRC [10].

Access to information is a study related to the processes involved in the organization, storage, retrieval, and discovery of information relevant to users' needs [11]. Access to information is the search for information from the existing collection of documents to find the most relevant information needed to query the user [12]. Because of the diversity of information available on the Internet, access information has an important role as a mechanism to ensure accurate information available.

The approach that can be implemented to overcome this difficulty is to implement a mechanism that can facilitate the computer to understand the meaning of search terms by users [1]. A way is needed to make words appearent in a web document which can be read and understood by machines or computers (machine readable data). Information retrieval that has this kind of ability as if it has artificial intelligence that can provide accurate answer for each consumers is required quiries. New technology in the web, known as the semantic web is required. Berners-Lee states that the semantic web "enables computers and people to work in better environments to provide certain information, which is structured and focused collaboration is the key to the implementation of the Semantic Web [13].

In the context of a university database, operational capabilities between components in the database will have multiple benefits. If two faculties from different universities conduct a similar research project, their profile information can be obtained from the websites of their respective universities. If the university adequately displays their data, researchers can find a partner or partners to carry out the research by only searching through faculty databases based on the term foaf: interest. Students can find the appropriate prospective supervisors with research that will be implemented. However, it was unclear how semantic web site or the technology used in the research community to build a system or to assist in improving the quality of search results made [14],[15].

Therefore the purpose of research proposals to provide components that can building semantic search engine. The work will be the foundation to facilitate semantic searches for the research community in the digital library system with a large-scale distribution. Subjects selected is research community in particular to assist in ensuring that the results obtained are based on the relevant requirements of users. The expected results in the research is an architecture that uses semantic technology that would improve semantic search on the web site for digital libraries and a semantic search mechanism that will provide better results and a combination or combinations of relevant search results and useful information to users. Researchers agree that the Semantic Web is a way to make a revolution in the internet world that would unite interactivity, information unity and artificial intelligence in a web portal [16],[17],[18]. With the concept of semantic web, information retrieval becomes more effective and relevant to consumer needs. Therefore a semantics portal model as a database in a community of faculty and in the field of research is needed.

\section{SEMANTIC WEB RESEARCH COMMUNITY}

Research on Semantic Web portal sites started quite early, in the era of 2000. Some design and implementation of web portals have been published in the paper Semantic research such as SEAL (semantic portal) from the Institute for Applied Informatics and Formal Description Method (AIFB), University of Karlsruhe has developed a semantic portal [19] and Portal Community Web Semantics [20]. Lausen et al, 2005 has provided many views in a choice of Semantic Web portal that has been published before 2005 [21]. Many research groups are now in charge or maintain a website 
portal of their group by using Semantic Web technologies. For example, Mindswap.org was used as the "the first OWLpowered Semantic Web site" [22] and the Semantic Mediawiki [23] was used to empower a number of portal sites, such as the Institute of Applied Informatics and Formal Description Methods (AIFB, aifb.kit.edu) and Tetherless World Constellation (tw.rpi.edu). Meanwhile, there are many web portals that semantic domain-specified that come from the winners of the "challenges of Semantic Web" [24] including the CS AKTive Space [25], Museum Finland [26],[17] Multimedia E-Culture demonstrator [27], HealthFinland [28] and TrialX [29].

Although the Semantic Web portal site is well developed, most of the sites are too difficult to be imitated by those who are not specialists. Visualization is one of the key components of a Semantic Web portal site [30],[31]. There are some general-purposed tools for visually presenting Semantic Web data, including linked data browsers such as Tabulator [32] and OpenLink Data Explorer (http://linkeddata.uriburner.com/ode), as well as data mashup tools such as sigma (aggregated instance description, sig.ma) and swoogle (aggregated semantic web term definition, swoogle.umbc.edu).

These tools render RDF triples directly via faceted filtering and customized rendering. SIMILE's Longwell (http://simile.mit.edu/wiki/Longwell_User_Guide) can be used to enable faceted browsing on RDF data, and Exhibit can further enable faceted visualization (e.g. map, timeline). It is notable that these tools differ from information visualization tools, which have more emphasis on rendering data into a graphical format.

\section{IV.ARCHITECTURE RC-FTSM}

RC-FTSM is a portal platform for the research community to view, find and visualize semantic data in a way that is user-friendly and meaningful. It can convert a time portal based on a relational database to Semantic Web a portal site. Fig. 2 shows the overall architecture, which contains the following main components:
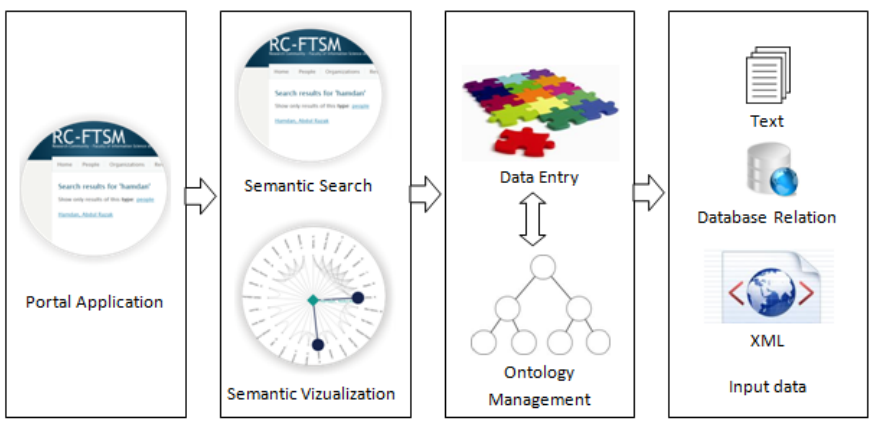

Fig. 2. RC-FTSM overall architecture

Semantic search: It enables a type-based search that can categorize federated RDF triples into different groups based on ontologies. It's based on Lucene (http://lucene.apache.org) and integrated with the ontologies portal that has been designated to provide searches based on type. For example, if the user enters "semantic web" as a search query into the RC-FTSM, they will receive RDF resources for that section or the words "semantic web," in which these resources are further categorized as a person, project, publications, presentations, and events.

Semantic Visualization: It displays all the limbs or in a university research group, or just members of the group who share a passion or interest in the same research.

Data Entry: Its main function is to convert input data in various formats into RDF triples.

Ontology Management: The main function of Ontology Management is to enable the creation of ontology-line tools, editing, browsing, mapping and clearing. RC-FTSM acts as a portal platform for semantic own website which can be used in any domain or application or a request to insert a display issue, describe and search for semantic data. At present, RC-FTSM has been used for a medium-sized research group to deal with topics about people, writing, grants, projects, presentations and research in semantics.

\section{Portal Ontology}

To create an appropriate ontology is a critical and important part of the RC-FTSM. Users need to create one or more database ontologies portal to change the existing relationship into RDF triples. It is useful to facilitate inquiries or the user's query, and display and portray the RDF data with meaning. There are several requirements in the production of generic ontologies for the RC-FTSM: 1) the ontology should reflect the database schema of its original dataset; 2) the key concepts or relationships identified from the questions or the most common user requests to be included in the ontology; 3) to enable interoperation, ontologies portal should try to use ontologies of existing sites, such as using foaf to represent the people (http://en.wikipedia.org/wiki/FOAF_software), using DOAP (http://en.wikipedia.org/wiki/Description_of_a_Project) to represent the project, using Bibontologi (bibliontology.com) to represent the production and use SIOC (sioc-project.org) to represent the online community, and; 4) Comply with the regulations or rules Linked Open Data (http://www.w3.org/ DesignIssues/LinkedData.html): using the HTTP URI to name the items, making the URI can be referred back and try to use the URI from the Linked Open Data the other as much as possible to assist the mapping tools.

Ontology is used in the research community portal to share some of the same URI and URI mapping related and consistent with the ontologies sites like foaf, SIOC, DOAP and BIBO. This allows us to link our data with several sets of Linked Open Data available, such as foaf, dbpedia and DBLP.

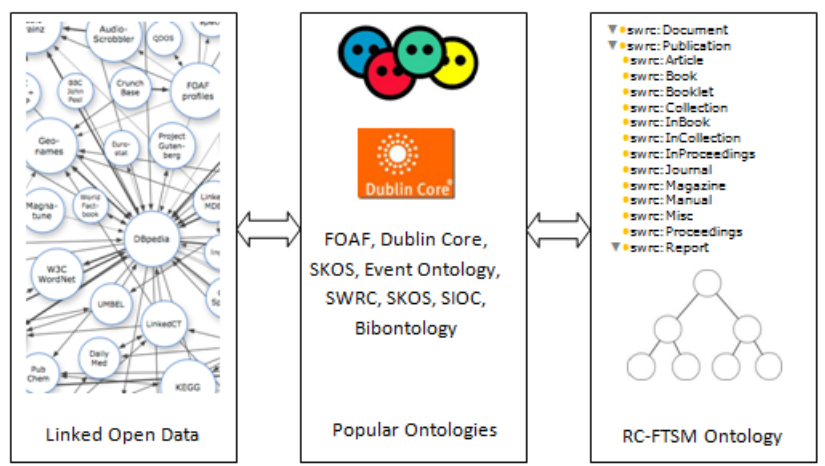

Fig. 3. The Ontology Framework Information Network 


\section{RESULT AND DISCUSSION}

From the semantics of the research community portal developed successfully to search for information based on criteria (research, publications, departments, organizations, events, and groups of researchers, etc.) in research work. Censorship of information search to find researchers who are experts in specific fields, such as information search to find experts in the field of semantic web. A visualization relationship between researcher and other researchers in a research community is shown in the Fig. 4 and Fig. 5

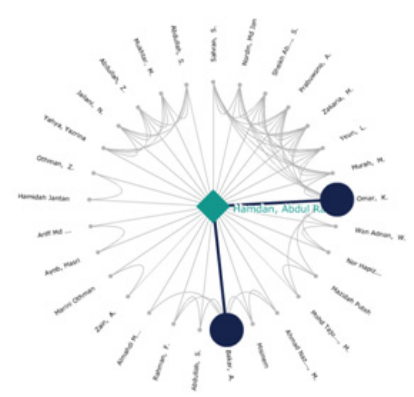

(a)

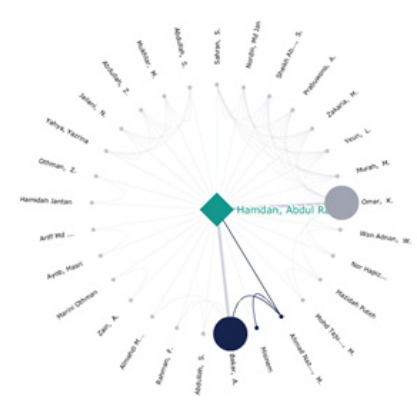

(b)
Fig. 4 Visualization Research Communities from Abdul Razak Hamdan

In fig. 4 , it is observable the visualization of university research community related to Abdul Razak Hamdan. In Fig.4 (a) it is shown that Abdul Razak Hamdan conducted research collaboration with 29 researchers and the most frequent research collaboration was with Khairuddin Omar and Azuraliza Abu Bakar was marked by circles from both increased. While at fig. 4 (b) is a visualization of Ahmad Nazri Mohd Zakree viewed from the research community in which Abdul Razak Hamdan can have the same two relationships.

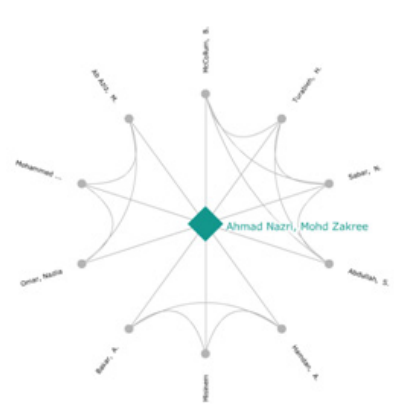

(a)

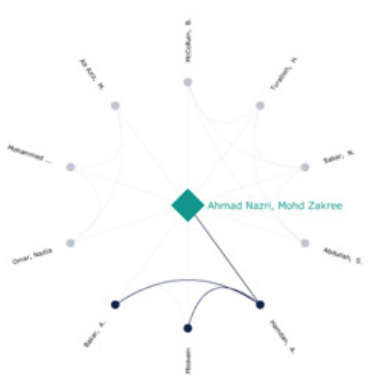

(b)
Fig.5 Visualization Research Community of Ahmad Nazri Mohd Zakree

In the visualization of fig. 5, it is apparent that university research community related to Zakree Ahmad Nazri Mohd. In fig. 5 (a) it emerged that Ahmad Nazri Mohd Zakree has done research collaboration with 10 researchers and the most frequent research collaboration equals to all researchers in the same field. While fig. 5 (b) is a visualization of Abdul Razak Hamdan, viewed from the research community of Ahmad Nazri Mohd Zakree which can have the two similar relationships.

\section{CONCLUSION}

Outlined solutions will strengthen the research information related to research, publications, departments, organizations, events, and groups of researchers. It will streamline the issuance process, making it easier for academic staff, support staff, and faculty itself to publish information of faculty and studies research information. This will provide end users with a better view of the structure of research at the university, allowing users to crosscommunication between faculty and study groups by using the search information. If this standard is used by other universities, it will help the joint venture between the faculty to streamline their efforts for obtaining research grants and create publications. It can also open doors to a whole new way to build relationships in the research.

\section{ACKNOWLEDGMENT}

The authors would like to acknowledge Universiti Kebangsaan Malaysia (Project code UKM-PTS-086-2010) for the financial support awarded for this research.

\section{REFERENCES}

[1] Berners-Lee. T., J. Hendler, O. Lassila, The Semantic Web, Scientific American, 2001.

[2] Anon, 2010. Internet Usage and World Population Statistics are for June 30, 2010, www.internetworldstats.com

[3] Anon, 2007. Web Server Survey. Netcraft.com; 2007. http://news.netcraft.com/archives/2007/12/29/december_2007_web_s erver survey. Html

[4] Lara.R, Han.S.K, Lausen.H, Stollberg.M, Ding.Y, Fensel. D.2004. An Evaluation of Semantic web Portals, Proceedings of the International Conference in Applied Computing (IADIS04), pp.23-26.

[5] Katy Börner, Luís M. A. Bettencourt, Mark Gerstein and Stephen M. Uzzo (Eds.) Knowledge Management and Visualization Tools in Support of Discovery. NSF Workshop Report, Indiana University, Los Alamos National Laboratory, Yale University, and New York Hall of Science (2009)

[6] Page. R.D.M. 2010. Enhanced display of scientific articles using extended metadata, Web Semantiks: Science, Services and Agents on the World Wide Web, vol. 8, pp. 190-195.

[7] Ding, L., Finin, T., Joshi, A., Pan, R., Cost, R.S., Sachs, J., Doshi, V., Reddivari, P., Peng, Y.2004. Swoogle: A search and metadata engine for the semantic web. In: Thirteenth ACM Conference on Information and Knowledge Management (CIKM 2004).

[8] d'Aquin, M., Baldassarre, C., Gridinoc, L., Angeletou, S., Sabou, M., Motta, E. 2007. Watson: A gateway for next generation semantic web applications. In: Poster session at the International Semantic Web Conference, ISWC 2007.

[9] Buitelaar, P., Eigner, T., Declerck, T. 2004. OntoSelect: A dynamic ontology library with support for ontology selection. In: Demo Session at the International Semantic Web Conference, ISWC 2004

[10] Sure, Y., et al. 2005. The SWRC Ontologi - Semantik Web for Research Communities, In Proceedings of the 12th Portuguese Conference on Artificial Intelligence - Progress in Artificial Intelligence (EPIA 2005). Springer. pp.218-231

[11] Ingwersen Peter.2002. information retrival. Taylor Graham. London John Borland (2007). A Smarter Web. Available: http://www.technologyreview.com/Infotech/18396/. Accessed: 20/07/09

[12] Langville. Amy N , Carl D. Meyer. 2006. Information Retrieval and Web Search. The Handbook of Linear Algebra. CRC Press. Charleston

[13] Gendarmi.D, Abbattista.F, Lanubile.F, Fostering knowledge evolution through community based participation, in: 16th International World Wide Web Conference, Banff, Alberta, Canada, 2007 
[14] Ding.H \& Sølvberg. I.T. 2006. Semantik Search in Peer-to-PeerBased Digital Libraries, European Digital Library, ERCIM News 66.pp 25-27

[15] Hidayat.R, Yahya.Y, Mohd Noah.S.A, 2011, The Semantik Portal for Supporting Research Community: a Review, Proceeding of the International Conference on Advanced Science, Engineering and Information Technology, pp. 327-330.

[16] Reynolds. Dave, Shabajee. Paul, Cayzer, Steve, 2004 Semantic information portals, Institute for Learning Technology Research, University of Bristol, 8-10 Berkeley Sq. Bristol, United Kingdom.

[17] Hyvonen, E., Junnila, J., Kettula, S., Mäkelä, E., Saarela, S. Salminen, M., Syreeni, A., Valo A., and Viljanen, K.2004. Finnish Museums on the Semantic Web. User's Perspective on MuseumFinland. Proceedings of Museums and the Web 2004 (MW2004), Archives \& Museum Informatics.

[18] Hyvonen, E., Ruotsalo, T., Häggström, T., Salminen, M., Junnila, M., Virkkilä, M., Haaramo, M., Kauppinen, T., Mäkelä, E., Viljanen, K. 2008.CultureSampo-Finnish culture on the semantic web. The vision and first results. In: Klaus Robering (Ed.), Information Technology for the Virtual Museum. LIT Verlag.

[19] Maedche, A., Staab, S., Stojanovic, N., Studer, R., and Sure, Y.: SEAL - A Framework for Developing SEmantic Web PortALs. In Proceedings of the 18th British National Conference on Databases: Advances in Databases, pp. 1--22 (2001)

[20] Staab, S., Angele, J., Decker, S., Erdmann, M., Hotho, A., Maedche, A., Schnurr, H., Studer, R., and Sure, Y.: Semantic community Web portals. Comput. Netw., pp. 473--491 (2006)

[21] Lausen,H., Ding, Y., Stollberg, M., Fensel, D., Hernandez, R., and Han,S.: Semantic web portals: state-of-the-art survey. Journal of Knowledge Management, vol. 9(5), pp. 40--49 (2005)

[22] Anon, 2004. Maryland Information and Network Dynamics Lab Semantic Web Agents Project. http://www.mindswap.org/first.shtml
[23] Krötzsch, M.: Semantic Media Wiki. http://semanticmediawiki.org /wiki/Semantic Media Wiki (2010)

[24] Bizer, C. and Maynard, D.: Semantic Web Challenge. http://challenge.semanticweb.org/ (2010)

[25] Schraefel, M.C., Shadbolt N.R., Gibbins, N., Glaser, H. and Harris, S.: CS AKTive Space: Representing Computer Science in the Semantic Web. Proceedings of the 13th International Conference on World Wide Web, pp. 384--392. ACM Press, New York (2004)

[26] Hyvönen, E., Junnila, M., Kettula, S., Mäkelä, E., Saarela, S., Salminen, M., Syreeni, A., Valo, A. and Viljanen, K.: Publishing Museum Collections on the Semantic Web: The Museumfinland Portal. Proceedings of the 13th international World Wide Web conference on Alternate track papers \& posters, pp. 418--419. ACM Press, New York (2004)

[27] Anon, 2008. MultimediaN N9C Eculture project: Multimedia ECulture demonstrator. http://eculture. multimedian.nl/index.shtml

[28] Suominen, O., Hyvönen, E., Viljanen, K. and Hukka, E.: HealthFinland-A National Semantic Publishing Network and Portal for Health Information. Web Semantics: Science, Services and Agents on the World Wide Web, 7(4), pp. 287--297 (2009)

[29] Anon, 2010. Applied Informatics, Inc.: TrialX: Enabling Patients to Find New Treatments. http://trialx.com/

[30] Cole, W. G., Shererta, D. D., Hsu, G., Fagan, L. M., and Carlson, R. W.: Semantic Visualization Of Oncology Knowledge Sources. Proc Annu Symp Comput Appl Med Care, pp. 67--71 (1995)

[31] Padgett, T., Maniquis, A., Hoffman, M., Miller, W., and Lautenschlager, J.: A Semantic Visualization Tool for Knowledge Discovery and Exploration in a Collaborative Environment. https://analysis.mitre.org/proceedings/Final_Papers_Files/171_Came ra Ready Paper.pdf (2005)

[32] Añon, 2005. (http://dig.csail.mit.edu/2005/ajar/ajaw/tab.html) 